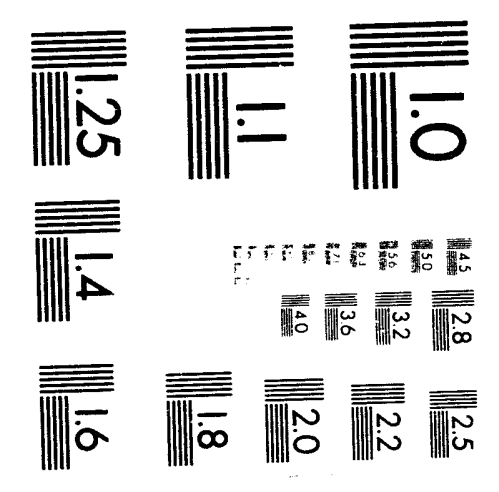



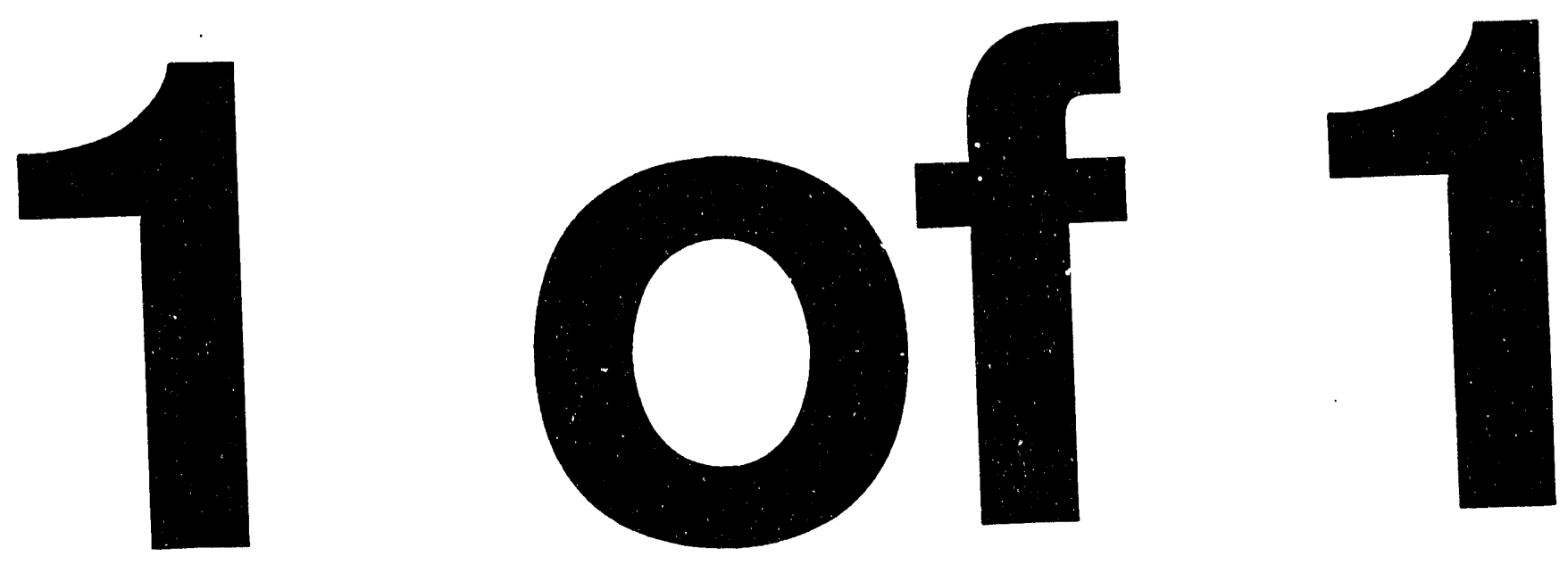


\section{WEDGE SCATTERING BY THE METHOD OF ITERATION}

By:

Dennis Holliday

Lester L. DeRaad, Jr.

Gaetan J. St-Cyr

July 1993

Prepared For:

Lawrence Livermore National Laboratory

P.O. Box 808

Livermore, CA 94550

Sponsored By:

Office of the Assistant

Secretary of Defense ( $\left.C^{3} I\right)$

Special Technology Office

The Pentagon

Room 3E-215

Washington, DC 20301-3040

\section{LOGICON}

\section{RDA}

R \& D ASSOCIATES, POST OFFICE BOX 92500, LOS ANGELES, CALIFORNIA 90009 6053 WEST CENTURY BOULEVARD - LOSANGELES, CA 90045 - TELEPHONE: 310 645-1122 
TABLE OF CONTENTS

Section

1

2

3

4

5

6
Page

INTRODUCTION . . . . . . . . . . . . . . . . 1

SOMMERFELD SOLUTIONS . . . . . . . . . . . . 3

WEDGE SCATTERING BY THE METHOD

OF ITERATION . . . . . . . . . . . . . . . . . . . . . 9

THE JVG WEDGE . . . . . . . . . . . . . . 22

SUMMARY OF CONCLUSIONS . . . . . . . . . . 28

LIST OF REFERENCES . . . . . . . . . . . . . . . . 29 


\section{LIST OF ILLUSTRATIONS}

Figure

Page

1 Geometry for wedge scattering usiı.g Sommerfeld Solutions (Section 2) . . . . . . . . . . . . . . . . . . . 4

2 Geometry for wedge scattering using Method of Iteration of the MFIE (Section 3) . . . . . . . . . . . . . . . . . . . . . 5

3 Rounded-off edge of the wedge $(R=\lambda / 16 ; \lambda=3 \mathrm{~cm})$. . . . . . . 10

$4 \quad$ V-pol surface current for a wedge with smoothed edge $R=\lambda / 16$. . . . . . . . . . . . . . . . . . . . . . . . 17

$5 \quad H$-pol surface current for a wedge with smoothed edge $R=\lambda / 16$. . . . . . . . . . . . . . . . . . . . . . . . 18

6

Boundary conditions ( $\mathrm{H}-\mathrm{pol}$ ) for a wedge with smoothed edge $R=\lambda / 16$. . . . . . . . . . . . . . . . . . . . . . . . 20

Boundary conditions (V-pol) for a wedge with smoothed edge $R=\lambda / 16$. . . . . . . . . . . . . . . . . . . . . . . . 21

8 Boundary conditions ( $\mathrm{H}$-pol) for the JVG wedge with $k_{\max }=1000 \mathrm{~m}^{-1}$ and $\delta y=0.5 \mathrm{~mm}$. . . . . . . . . . . . . . . 26

$9 \quad$ Boundary conditions (V-pol) for the JVG wedge with $k_{\max }=1000 \mathrm{~m}^{-1}$ and $\delta y=0.5 \mathrm{~mm}$. . . . . . . . . . . . . . . 27 


\section{SECTION 1 INTRODUCTION}

We have investigated scattering from the classic wedge and have shown that the method of iteration of the surface current integral equation predicts currents and backscattered fields that are good approximations to the Sommerfeld solution. The method of iteration has also been applied to truncated wedges on flat surfaces with the result that the scattering from this wedge is seen to be very much different from the Sommerfield solution. These results and their implications for ocean backscatter are reported herein.

Sommerfeld solved the problem of electromagnetic scattering from a perfectly conducting wedge in his classic 1896 paper on diffraction (Ref. 1). Since that time, there have been a number of investigations (Ref. 2 and references therein) of the analytic properties of his solutions. A succinct review can be found in Reference 2. These solutions to wedge scattering form the basis of scattering from sharp, wedgelike objects as treated by the Uniform Geometrical Theory of Diffraction (UGTD) (Ref. 3). Scattering from wedgelike objects on the ocean's surface is sometimes (Ref. 4-5) invoked as an important scattering mechanism to help explain the large experimental values of the polarization ratio $\left(\sigma_{H H} / \sigma_{V V}\right)$ observed at low grazing angles (Ref. 6). These experimental values vary from about $-20 \mathrm{~dB}$ to near $0 \mathrm{~dB}$. Theories based on Bragg resonance backscatter predict far lower values than these, typically, about $-40 \mathrm{~dB}$.

Sommerfeld's solutions for the total electromagnetic fields are in terms of contour integrals in the complex plane. The asymptotic forms for the diffracted fields are cylindrical waves generated by line sources located at the edge of the wedge (Ref. 7). These asymptotic solutions have played an important role in the development of Keller's original geometrical theory of diffraction (GTD) (Ref. 8). Some shortcomings of Keller's theory, particularly dealing with the transition between shadowed and unshadowed regions, were overcome by Kouyoumjian and Pathak in their uniform theory of diffraction (UTD) (Ref. 9). Many of the same issues can be dealt with using the theory of equivalent currents (Ref. 10). All of these theories are improvements to the theory of geometric optics (GO) and all exploit the asymptotic behavior of Sommerfeld's solutions when applied to scattering from edges. It is important to keep in mind that all these theories are applicable in the high frequency limit where the diffraction process can be viewed as being approximately local. 
One application of these extensions to GO has been to calculate the scattering produced by wedgelike objects on the ocean's surface. Lyzenga et al. (Ref. 4) suggested a twodimensional wedge scattering model based on the theory of equivalent currents (Ref. 10). A three-dimensional model is discussed in Reference 5 (hereafter referred to as the JVG wedge), where the scattered field is calculated by UGTD (Ref. 3). This latter paper was one of the motivations for us to investigate wedge scattering and is the source of the particular parameters we have considered. It is to be noted that the height of the JVG wedge is small on the scale of the wavelength of the incident radiation and that the polarization ratio at 85 degrees incidence is reported to be about $-22 \mathrm{~dB}$. This polarization ratio is significantly different from the $-42 \mathrm{~dB}$ we have calculated because, as we will show explicitly, the JVG wedge does not scatter as a classical Sommerfeld wedge, which is the implicit underlying assumption of Reference 5 .

The outline of this paper is as follows. In Section 2 we review the Sommerfeld solution, and we show in Section 3 that a very accurate approximation to this solution can be obtained by the method of iteration. A calculation of the backscattered fields for a simplified version of the JVG wedge is discussed in Section 4 . Section 5 summarizes the main conclusions in this paper. 


\section{SECTION 2 \\ SOMMERFELD SOLUTIONS}

The geometry for wedge scattering with an incident plane wave is shown in Figure 1 as adapted from Reference 2. The cartesian coordinates are $(x, y, z)$, where $\widehat{\mathbf{x}}$ is to the right, $\widehat{\mathbf{y}}$ is up, and $\widehat{\mathbf{z}}$ is out of the paper. Note that this is a slightly different coordinate system than that employed for the calculations discussed in Section 3 [cf. Figure 2.] The enclosed angle of the wedge is $2 \Omega$, and the backscatter direction relative to the $\mathrm{x}$-axis is $\phi_{0}$, so that the grazing angle $\theta_{g}$ relative to the upper face of the wedge is

$$
\theta_{g}=\phi_{0}-\Omega
$$

A parameter characteristic of the wedge is

$$
\nu=2\left(1-\frac{\Omega}{\pi}\right) \text {. }
$$

For the numerical calculations discussed below, we set

$$
\begin{aligned}
\theta_{g} & =27^{\circ}, \\
\Omega & =68^{\circ} .
\end{aligned}
$$

Notice that for these parameters, the lower face of the wedge is in shadow. The calculations will be done at $X$-band, where the incident radiation has a wavelength $\lambda$ of $3 \mathrm{~cm}$.

For our purposes, we will consider the asymptotic form of the diffracted fields for which $\kappa \rho \gg 1$. Here, $\kappa$ is the wavenumber of the incident radiation:

$$
\kappa=\frac{2 \pi}{\lambda}
$$

where $\rho$ is the radial distance in a cylindrical coordinate system centered at the edge of the wedge (Figure 1). 


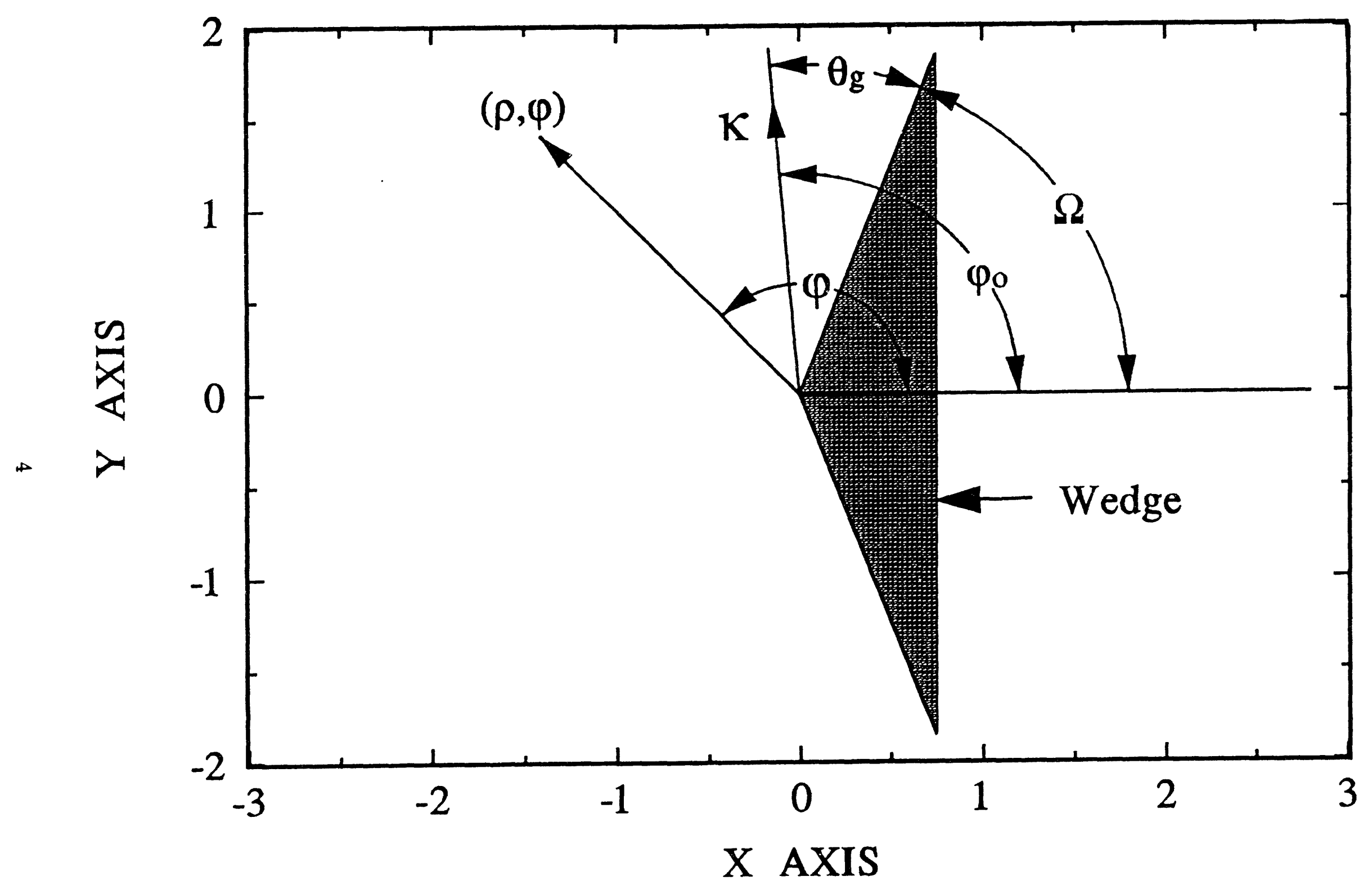

Figure 1: Geometry for wedge scattering using Sommerfeld Solutions (Section 2). 


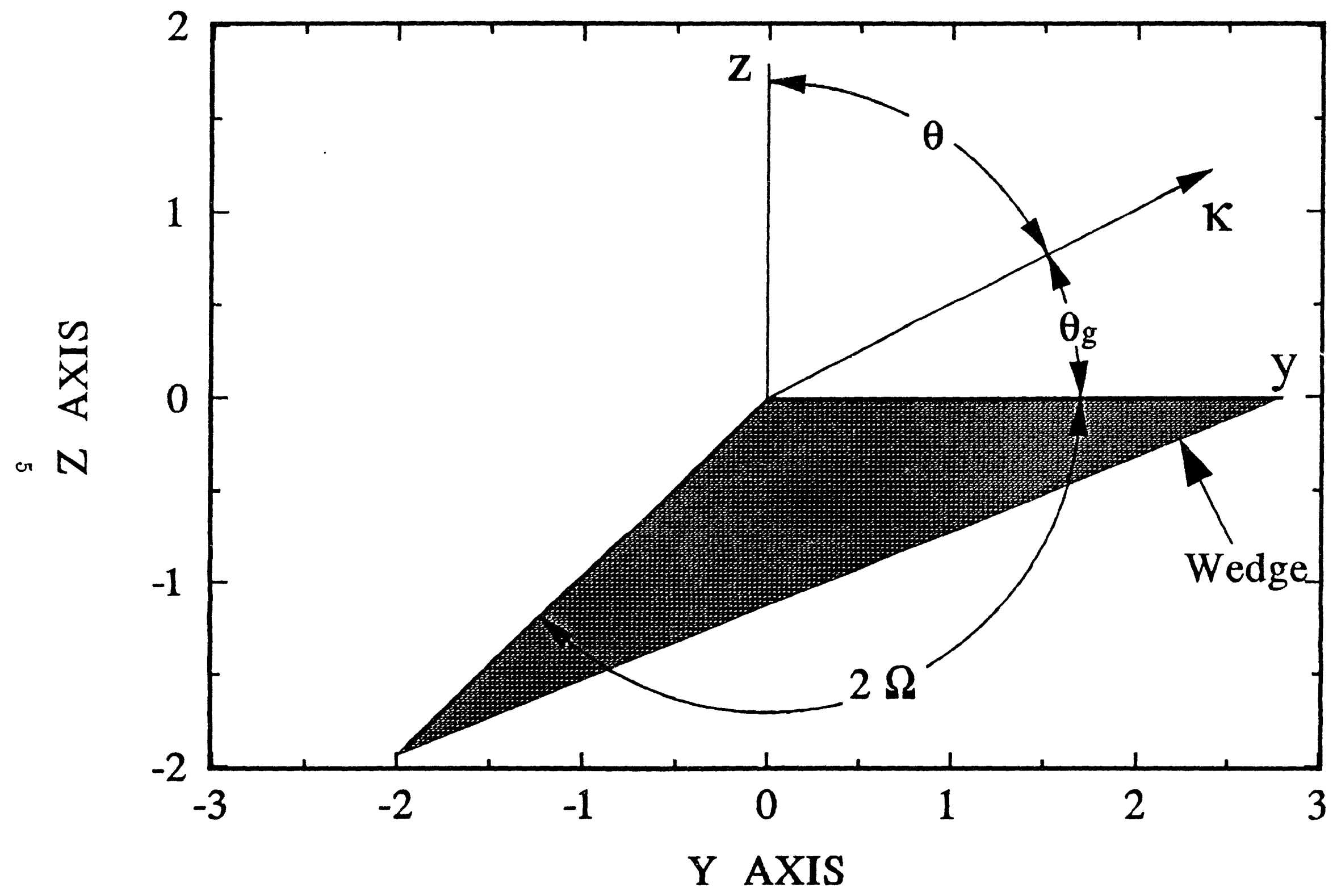

Figure 2: Geometry for wedge scattering using Method of Iteration of the MFIE (Section 3). 
For vertical polarization, or V-pol, the magnetic field is parallel to the edge of the wedge. The asymptotic form of the diffracted magnetic field away from geometrical optics boundaries is (Ref. 2):

$$
B_{z}^{d} \approx \frac{e^{i\left(\kappa \rho+\frac{1}{4} \pi\right)}}{\sqrt{2 \pi \kappa \rho}} \frac{1}{\nu} \sin \frac{\pi}{\nu}\left\{\left(\cos \frac{\pi}{\nu}-\cos \frac{\phi-\phi_{0}}{\nu}\right)^{-1}+\left(\cos \frac{\pi}{\nu}+\cos \frac{2 \pi-\phi-\phi_{0}}{\nu}\right)^{-1}\right\},
$$

where the subscript $z$ refers to the $\mathbf{z}$ direction, normal to the paper in Figure 1.

Equation (5) can be used to calculate the current on the surface of the wedge far from the edge. Defining $\widehat{\mathbf{n}}$ to be the unit vector normal to the surface and pointing away from the wedge, the surface current $\mathcal{J}=\widehat{\mathbf{n}} \times \mathbf{B}$, is:

$$
\begin{array}{ll}
\mathcal{J}=\hat{\boldsymbol{\rho}}\left[2 e^{-i \kappa \rho \cos \theta_{g}}+B_{z}^{d}(\phi=\Omega)\right] & \text { for the upper face } \\
\mathcal{J}=-\widehat{\rho} B_{z}^{d}(\phi=2 \pi-\Omega) & \text { for the lower face. }
\end{array}
$$

Note that $\hat{\boldsymbol{\rho}}$ points directly away from the edge. The surface currents, Eqs. (6) and (7), include both the incident and the reflected fields. Equation (5) may also be used to calculate the backscattered field, as will be discussed at the end of this section.

For horizontal polarization, H-pol, the electric field is parallel to the edge of the wedge. The asymptotic form of the diffracted electric field away from geometrical optics boundaries is (Ref. 2):

$$
E_{z}^{d} \approx \frac{e^{i\left(\kappa \rho+\frac{1}{4} \pi\right)}}{\sqrt{2 \pi \kappa \rho}} \frac{1}{\nu} \sin \frac{\pi}{\nu}\left\{\left(\cos \frac{\pi}{\nu}-\cos \frac{\phi-\phi_{0}}{\nu}\right)^{-1}-\left(\cos \frac{\pi}{\nu}+\cos \frac{2 \pi-\phi-\phi_{0}}{\nu}\right)^{-1}\right\} .
$$

To calculate the surface current, we first calculate the magnetic field using Maxwell's equation

$$
\mathbf{B}=-\frac{i}{\kappa} \nabla \times \mathbf{E}
$$

Since $\mathbf{E}$ is in the $\mathbf{z}$ direction, normal to the paper in Figure 1, $\mathbf{B}$ is in the plane of the paper and consists of two components. The $\hat{\boldsymbol{\rho}}$ component is:

$$
B_{\rho}^{d}=-i \frac{e^{i\left(\kappa \rho+\frac{1}{4} \pi\right)}}{\sqrt{2 \pi \kappa \rho}} \frac{1}{\kappa \rho} \frac{1}{\nu^{2}} \sin \frac{\pi}{\nu}\left\{-\frac{\sin \frac{\phi-\phi_{0}}{\nu}}{\left[\cos \frac{\pi}{\nu}-\cos \frac{\phi-\phi_{0}}{\nu}\right]^{2}}+\frac{\sin \frac{2 \pi-\phi-\phi_{0}}{\nu}}{\left[\cos \frac{\pi}{\nu}+\cos \frac{2 \pi-\phi-\phi_{0}}{\nu}\right]^{2}}\right\},
$$


and the $\widehat{\phi}$ component is:

$$
B_{\phi}^{d}=-\frac{e^{i\left(\kappa \rho+\frac{1}{4} \pi\right)}}{\sqrt{2 \pi \kappa \rho}} \frac{1}{\nu} \sin \frac{\pi}{\nu}\left\{\left(\cos \frac{\pi}{\nu}-\cos \frac{\phi-\phi_{0}}{\nu}\right)^{-1}-\left(\cos \frac{\pi}{\nu}+\cos \frac{2 \pi-\phi-\phi_{0}}{\nu}\right)^{-1}\right\}
$$

On the surface of the wedge, the unit vector $\widehat{\phi}$ is parallel to the normal unit vector $\widehat{\mathbf{n}}$, so that $B_{\phi}^{d}$ makes no contribution to the induced surface current $\mathcal{J}=\widehat{\mathbf{n}} \times \mathbf{B}$. On the other hand, the unit vector $\hat{\boldsymbol{\rho}}$ is perpendicular to $\widehat{\mathbf{n}}$ so that $B_{\rho}^{d}$ provides the sole contribution to $\mathcal{J}:$

$$
\begin{array}{ll}
\mathcal{J}=\widehat{\mathbf{z}}\left[2 \sin \theta_{g} e^{-i \kappa \rho \cos \theta_{g}}-B_{\rho}^{d}(\phi=\Omega)\right] & \text { for the upper face } \\
\mathcal{J}=\widehat{\mathbf{z}} B_{\rho}^{d}(\phi=2 \pi-\Omega) & \text { for the lower face. }
\end{array}
$$

Comparing Eqs. (12) and (13) to Eqs. (6) and (7), we observe that the induced surface current $\mathcal{J}$ is perpendicular to the edge of the wedge for vertical polarization and parallel to the edge of the wedge for horizontal polarization.

The comnponent $B_{\phi}^{d}$ in Eq. (11), along with $B_{z}^{d}$ in Eq. (5), can now be used to calculate a quantity that is proportional to the backscattered field. We calculate, for each polarization:

$$
\mathcal{B}=\int d \ell \mathbf{B}_{0} \cdot(\mathcal{J} \times \kappa) e^{-i \kappa \rho(\ell) \cos \left(\phi(\ell)-\phi_{0}\right)},
$$

where $d \ell$ is an increment along the surface, $\phi=\Omega$ for the upper face and $\phi=2 \pi-\Omega$ for the lower face and, in the cartesian coordinate system of Figure 1,

$$
\begin{aligned}
& \boldsymbol{\kappa}=\kappa\left(\cos \phi_{0}, \quad \sin \phi_{0}, \quad 0\right), \\
& \text { H-pol: } \quad \mathbf{B}_{0}=(0,0,1) \text {, } \\
& \text { V-pol: } \quad \mathbf{B}_{0}=\left(\begin{array}{lll}
-\sin \phi_{0}, & \cos \phi_{0}, & 0
\end{array}\right) \text {. }
\end{aligned}
$$

By means of the Stratton-Chu equation, one can show that $\mathcal{B}$ can be related to the backscattered magnetic field by

$$
\mathbf{B}_{0} \cdot \mathbf{B}_{S}\left(\rho, \phi_{0}\right)=-\frac{i}{2} \frac{e^{i\left(\kappa \rho+\frac{1}{4} \pi\right)}}{\sqrt{2 \pi \kappa \rho}} \mathcal{B}
$$


The predicted values for $\mathcal{B}$, for each polarization, are then [using Eqs. (11) and (5)]:

$$
\begin{gathered}
\mathcal{B}^{H}=-2 i \frac{1}{\nu} \sin \frac{\pi}{\nu}\left\{\left(\cos \frac{\pi}{\nu}-1\right)^{-1}-\left(\cos \frac{\pi}{\nu}+\cos \frac{2\left(\pi-\phi_{0}\right)}{\nu}\right)^{-1}\right\} \\
\mathcal{B}^{V}=2 i \frac{1}{\nu} \sin \frac{\pi}{\nu}\left\{\left(\cos \frac{\pi}{\nu}-1\right)^{-1}+\left(\cos \frac{\pi}{\nu}+\cos \frac{2\left(\pi-\phi_{0}\right)}{\nu}\right)^{-1}\right\} .
\end{gathered}
$$

For the numerical values given in Eq. (3), we have

$$
\begin{aligned}
& \mathcal{B}^{H}=-9.079 \times 10^{-2} i \\
& \mathcal{B}^{V}=-1.115 i .
\end{aligned}
$$

This implies a polarization ratio of $-21.8 \mathrm{~dB}$. 


\section{SECTION 3 \\ WEDGE SCATTERING BY THE \\ METHOD OF ITERATION}

In this section, we calculate the surface currents and backscattered fields for the wedge shown in Figure 2 by means of iteration of the magnetic field integral equation (MFIE). Note that this is the same wedge as the one described in Figure 1 and Eq. (3). The enclosed angle of the wedge is still $2 \Omega=136^{\circ}$, and the grazing angle, relative to the upper face of the wedge, is still $\theta_{g}=27^{\circ}$. Only the coordinate system has been redefined. The upper face coincides with the $y$-axis, while the lower face is characterized by a slope $s_{0}$. Since we are employing the MFIE, the surface must possess continuous height and slope variations. Consequently, we can not directly use the slope discontinuity exhibited by the wedge in Figure 2. Instead, we introduce a sharply peaked surface to smoothly connect the two flat planes. The surface is expressed in terms of the height $\eta(y)$ :

$$
\eta(y)=\left\{\begin{array}{lc}
0, & y \geq y_{0} \\
y_{0} s_{0}\left(\frac{y-y_{0}}{2 y_{0}}\right)^{3}\left(2+\frac{y-y_{0}}{2 y_{0}}\right), & -y_{0} \leq y \leq y_{0}, \\
s_{0} y, & y \leq-y_{0},
\end{array}\right\}
$$

where $y_{0}$ is

$$
y_{0}=\frac{3}{4} s_{0} R
$$

and $1 / R$ is the maximum curvature. This surface has continuous zeroth, first and second derivatives; it is, therefore, a Lyapunov surface. Figure 3 shows the connecting surface compared to the sharp wedge for $R=\lambda / 16, s_{0}=\tan \left(44^{\circ}\right)$ and $\lambda=3 \mathrm{~cm}$. The maximum curvature for this case is $533 \mathrm{~m}^{-1}$.

The quantities we calculate, $\mathbf{j}(y)$, are related to the surface currentis and are defined as

$$
\mathbf{j}(y)=\mathbf{n}(y) \times \mathbf{B}(y)
$$




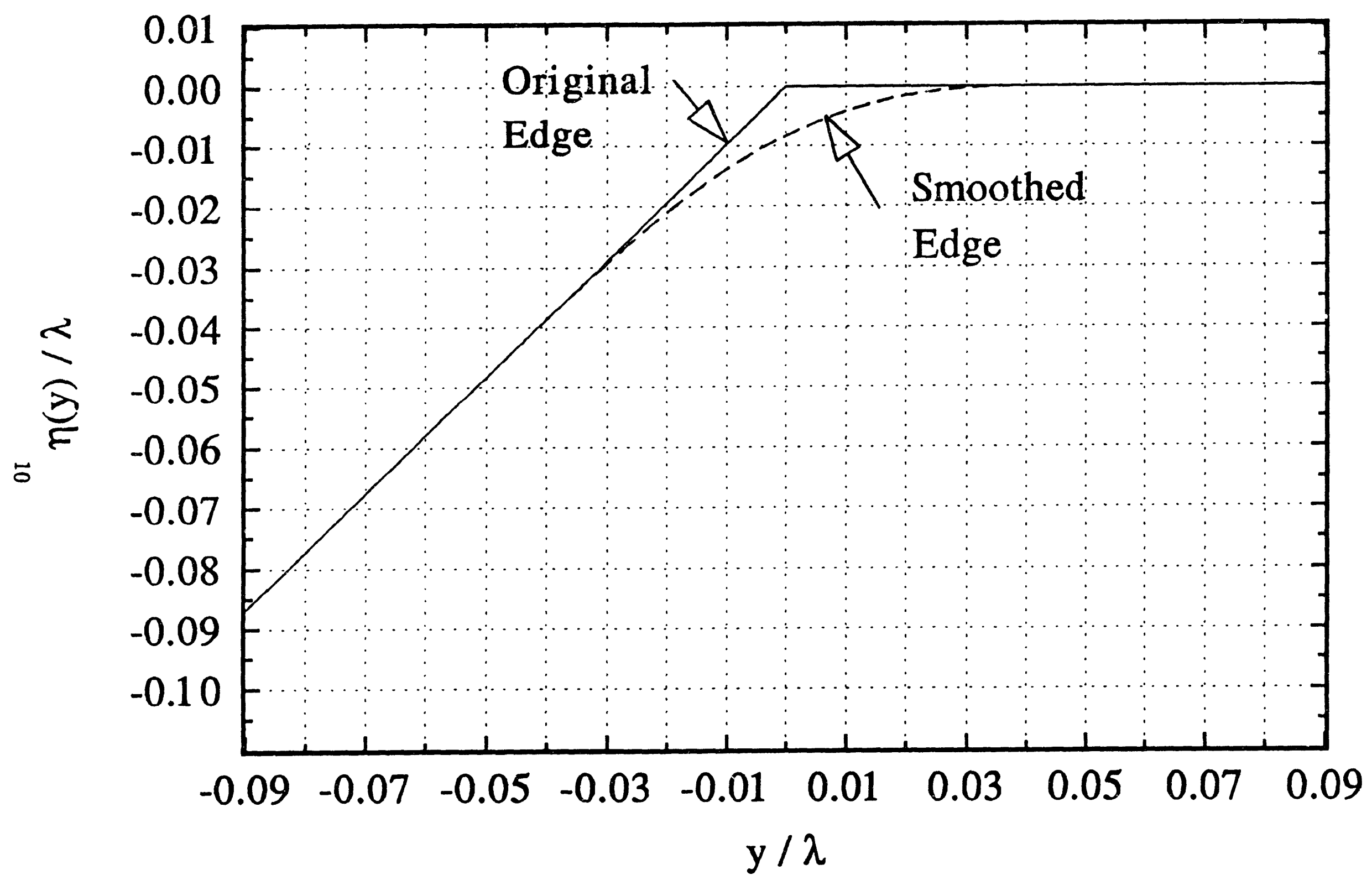

Figure 3: Rounded-off edge of the wedge $(R=\lambda / 16 ; \quad \lambda=3 \mathrm{~cm})$. 
where $\mathbf{B}(y)$ is the total magnetic field on the surface at horizontal coordinate $y$, and $\mathbf{n}(y)$ is the (unnormalized) normal to the surface at this point:

$$
\mathbf{n}(y)=(0,-s(y), 1)
$$

where $s(y)$ is the slope

$$
s(y)=\frac{d}{d y} \eta(y)
$$

Convenient representations for the surface currents are

$$
\begin{array}{ll}
\text { V-pol: } & \mathbf{j}(y)=(0,1, s(y)) J^{V}(y) e^{-i \kappa(y \sin \theta+\eta(y) \cos \theta)}, \\
\text { H-pol: } & \mathbf{j}(y)=(1,0,0) \quad J^{H}(y) e^{-i \kappa(y \sin \theta+\eta(y) \cos \theta)} .
\end{array}
$$

The incidence angle relative to the forward plane of the wedge is $\theta=63^{\circ}$ [see Fig. 2], and the backscatter wavenumber vector is

$$
\kappa=\kappa(0, \sin \theta, \cos \theta)
$$

The quantities $J^{V}(y)$ and $J^{H}(y)$ will be referred to as surface currents in V-pol and H-pol, respectively.

The MFIE for the surface currents are the following (Ref. 11):

$$
\begin{aligned}
& J^{V}(y)=2+\int d y^{\prime} G^{V}\left(y, y^{\prime}\right) J^{V}\left(y^{\prime}\right) \\
& J^{H}(y)=2(\cos \theta-\sin \theta s(y))+\int d y^{\prime} G^{H}\left(y, y^{\prime}\right) J^{H}\left(y^{\prime}\right), \\
& G^{V}\left(y, y^{\prime}\right)= \frac{i}{2} \kappa\left[\eta(y)-\eta\left(y^{\prime}\right)+s\left(y^{\prime}\right)\left(y^{\prime}-y\right)\right] \frac{1}{\gamma} H_{1}(\kappa \gamma) \\
& \cdot e^{-i \kappa\left[\left(y^{\prime}-y\right) \sin \theta+\left(\eta\left(y^{\prime}\right)-\eta(y)\right) \cos \theta\right]} \\
& G^{H}\left(y, y^{\prime}\right)=\frac{i}{2} \kappa\left[\eta(y)-\eta\left(y^{\prime}\right)+s(y)\left(y^{\prime}-y\right)\right] \frac{1}{\gamma} H_{1}(\kappa \gamma) \\
& \cdot e^{-i \kappa\left[\left(y^{\prime}-y\right) \sin \theta+\left(\eta\left(y^{\prime}\right)-\eta(y)\right) \cos \theta\right]}
\end{aligned}
$$

where $\gamma$ is the distance between the two points on the surface 
To solve the MFIE for the currents, we use the result that the asymptotic values of the surface current are known. In particular, we assume

$$
\begin{array}{ll}
y>y_{+}: & J^{V}(y)=2, \\
J^{H}(y)=2 \cos \theta \\
J^{V}(y)=0, \\
J^{H}(y)=0 .
\end{array}
$$

As can be seen from the asymptotic expressions for the wedge solutions, the assumed values are easy to obtain in H-pol and very difficult to obtain in V-pol. Typically, we used

$$
\begin{aligned}
& y_{+}=16 \mathrm{~m} \\
& y_{-}=-8 \mathrm{~m} .
\end{aligned}
$$

The fact that the V-pol results are somewhat inaccurate outside this domain seems to affect the scattered field or the calculated current very little. Including the contributions from the distant flat plates, which are given by Eqs. (33) and (34), the actual integral equations that we solve by iteration on the interval $y_{-}<y<y_{+}$, are

$$
\begin{aligned}
& J^{V}(y)=2+i \kappa \eta(y) e^{i \kappa \cos \theta \eta(y)} T_{V}(y)+\int_{y_{-}}^{y_{+}} d y^{\prime} G^{V}\left(y, y^{\prime}\right) J^{V}\left(y^{\prime}\right), \\
& J^{H}(y)=2(\cos \theta-\sin \theta s(y))+i \cos \theta e^{i \kappa \cos \theta \eta(y)}\left[\kappa \eta(y) T_{V}(y)+s(y) T_{H}(y)\right] \\
&+\int_{y_{-}}^{y_{+}} d y^{\prime} G^{H}\left(y, y^{\prime}\right) J^{H}\left(y^{\prime}\right) .
\end{aligned}
$$

The additional functions introduced here are

$$
\begin{aligned}
& T_{V}(y)=\int_{y_{+}}^{\infty} d y^{\prime} \frac{1}{\tilde{\gamma}} H_{1}(\kappa \tilde{\gamma}) e^{-i \kappa \sin \theta\left(y^{\prime}-y\right)} \\
& T_{H}(y)=\kappa \int_{y_{+}}^{\infty} d y^{\prime} \frac{y^{\prime}-y}{\widetilde{\gamma}} H_{1}(\kappa \tilde{\gamma}) e^{-i \kappa \sin \theta\left(y^{\prime}-y\right)}
\end{aligned}
$$

where

$$
\widetilde{\gamma}=\left[\left(y^{\prime}-y\right)^{2}+\eta^{2}(y)\right]^{\frac{1}{2}}
$$

Reference 11 contains estimates for these functions that are accurate at low grazing angles. However, for the geometrical convention used here, the grazing angle (relative to the 
forward face) is 27 degrees $\left(\theta=63^{\circ}\right)$, for which a different asymptotic expansion is more appropriate. In particular, we employ

$$
\begin{aligned}
& T_{V}(y) \sim \frac{1}{u} H_{0}(d) e^{-i u \sin \theta}-\frac{i}{g} e^{i f} \sum_{k=0}^{1}\left[\frac{d}{d u} \frac{i}{g}\right]^{k} F_{V}(u), \\
& T_{H}(y) \sim H_{0}(d) e^{-i u \sin \theta}+\frac{\sin \theta}{g} e^{i f} \sum_{k=0}^{2}\left[\frac{d}{d u} \frac{i}{g}\right]^{k} F_{H}(u),
\end{aligned}
$$

where

$$
\begin{aligned}
u & =\kappa\left(y_{+}-y\right), \\
d & =\left[u^{2}+\kappa^{2} \eta^{2}(y)\right]^{\frac{1}{2}}, \\
f & =d-u \sin \theta, \\
g & =\frac{u}{d}-\sin \theta, \\
F_{V}(u) & =\left(\frac{1}{u^{2}}+\frac{i \sin \theta}{u}\right) H_{0}(d) e^{-i d}, \\
F_{H}(u) & =H_{0}(d) e^{-i d} .
\end{aligned}
$$

As noted above, the lower face of the wedge is in shadow. From the point of view of the integral equations, shadowing occurs because, for the lower face $(y<0)$, there is a stationary phase point $y_{S P}$ on the upper face for the multiple scattering integral. The location of this stationary phase point is

$$
y_{S P}=\left(s_{0} \tan \theta-1\right)|y| .
$$

The above asymptotic expressions are accurate only if the stationary phase point does not lie in the region of integration implied by the definitions of $T_{V}$ and $T_{H}$, that is,

$$
y_{S P} \ll y_{+} .
$$

Clearly, for fixed $y_{+}, y_{-}$has to be restricted. The values we use, Eq. (35), satisfy Eq. (50). The other point to notice here is that, as the grazing angle decreases, $y_{+}$has to increase for fixed $y_{-}$.

The form of the integral equations [Eqs. (36) and (37)] is then

$$
J(y)=D(y)+\int_{y_{-}}^{y_{+}} d y^{\prime} G\left(y, y^{\prime}\right) J\left(y^{\prime}\right)
$$


Defining $J_{N}(y)$ as the solution for the total induced current at the end of $N$ iterations, we determine $J_{N+1}(y)$ by the equation

$$
J_{\mathrm{N}+1}(y)=D(y)+\int_{y_{-}}^{y_{+}} d y^{\prime} G\left(y, y^{\prime}\right) J_{N}\left(y^{\prime}\right)
$$

where the starting point $J_{0}(y)$ of the iteration process is

$$
\begin{aligned}
& \text { V-pol: } \\
& \text { H-pol: }
\end{aligned} \quad J_{0}(y)=\left\{\begin{array}{l}
2, \\
2(\cos \theta-s(y) \sin \theta) .
\end{array}\right.
$$

The procedure in Eq. (52) is continued until the maximum value of the absolute change in the current, $\delta \mathrm{J}$, is less than $10^{-6}$ everywhere in the computational domain,

$$
\left|\delta J_{N}\right|=\max \left|J_{N+1}(y)-J_{N}(y)\right|<10^{-6}
$$

Ten iterations or less were required for each of the three calculations described here.

We have calculated the surface currents for both polarizations as well as the backscattered fields Eq. (14) for the parameters mentioned above: that is, $\theta=63^{\circ}$ and $\lambda=3 \mathrm{~cm}$. Three values of $R$ were used: $R=\lambda / 4, \lambda / 8$ and $\lambda / 16$. The typical sampling interval for the calculations was $1 \mathrm{~mm}(\lambda / 30)$, although the peaked region for $R=\lambda / 16$ had a sampling interval of $1 / 2 \mathrm{~mm}$. Table 1 shows the backscattered fields computed by the iteration method for the three values of $R$, as well as those computed using the Sommerfeld Solutions. As $R$ decreases (i.e.: as the edge curvature increases), both the amplitude and phase of the B-fields approach those calculated by the Sommerfeld Solutions. Thus, smooth but sufficiently highly peaked surfaces are shown to closely approximate infinitely sharp surfaces. 
Table 1: Backscattered Fields By Iteration

\begin{tabular}{|c|c|c|c|}
\hline \multicolumn{2}{|c|}{$\mathbf{R}$} & Amplitude & $\begin{array}{c}\text { Phase } \\
\text { ( Degrees) }\end{array}$ \\
\hline$\lambda / 4$ & $V$ & 0.608 & -116 \\
& $H$ & $4.87 \times 10^{-2}$ & -135 \\
\hline$\lambda / 8$ & $V$ & 0.931 & -98 \\
& $H$ & $7.13 \times 10^{-2}$ & -102 \\
\hline$\lambda / 16$ & $V$ & 1.062 & -93 \\
& $H$ & $8.28 \times 10^{-2}$ & -94 \\
\hline Sommerfeld & $V$ & 1.115 & -90 \\
\hline
\end{tabular}

The convergence of the integral equations as a function of the number of iterations is illustrated in Table 2 for $\mathrm{V}$-pol and in Table 3 for $\mathrm{H}$-pol for $R=\lambda / 16$. The convergence is slightly more rapid in V-pol than in $\mathrm{H}$-pol. As mentioned above, we continued the iteration process beyond what is shown in Tables 2 and 3 until the $10^{-6}$ limit was attained (10 iterations for this case). Iterations beyond those in the Tables show that the scattered fields did not change sufficiently and the $\delta J$ simply continued to decrease. There was no indication of instability in the iteration process at this level of numerical accuracy. 
Table 2: V-Pol Convergence

\begin{tabular}{|c|c|c|c|}
\hline $\mathbf{N}$ & $\boldsymbol{\delta J}$ & $\begin{array}{c}\text { Backscattered Field } \\
\text { Amplitude }\end{array}$ & $\begin{array}{c}\text { Phase } \\
\text { ( Degrees) }\end{array}$ \\
\hline 0 & - & 0.494 & -95 \\
1 & $1.2 \times 10^{-1}$ & 0.978 & -96 \\
2 & $2.1 \times 10^{-2}$ & 1.046 & -93 \\
3 & $3.9 \times 10^{-3}$ & 1.066 & -93 \\
4 & $7.6 \times 10^{-4}$ & 1.061 & -93 \\
5 & $1.5 \times 10^{-4}$ & 1.062 & -93 \\
6 & $2.9 \times 10^{-5}$ & 1.062 & -93 \\
\hline
\end{tabular}

Table 3: H-Pol Convergence

\begin{tabular}{|c|c|c|c|}
\hline $\mathbf{N}$ & $\delta \mathrm{J}$ & $\begin{array}{c}\text { Backscattered Field } \\
\text { Amplitude }\end{array}$ & $\begin{array}{c}\text { Phase } \\
\text { ( Degrees) }\end{array}$ \\
\hline$n$ & - & $4.94 \times 10^{-1}$ & -95 \\
1 & $2.1 \times 10^{-1}$ & $4.28 \times 10^{-2}$ & -136 \\
2 & $3.8 \times 10^{-2}$ & $1.08 \times 10^{-1}$ & -94 \\
3 & $6.8 \times 10^{-3}$ & $8.84 \times 10^{-2}$ & -92 \\
4 & $1.3 \times 10^{-3}$ & $8.38 \times 10^{-2}$ & -93 \\
5 & $2.6 \times 10^{-4}$ & $8.29 \times 10^{-2}$ & -94 \\
6 & $4.9 \times 10^{-5}$ & $8.28 \times 10^{-2}$ & -94 \\
\hline
\end{tabular}

Figures 4 and 5 show the V-pol and H-pol induced surface currents for $R=\lambda / 16$. The H-pol current decreases very rapidly in the shadowed region, while the $V$-pol current decreases much more slowly. In fact, at $y=y_{-}$, we still have a rather large value of 


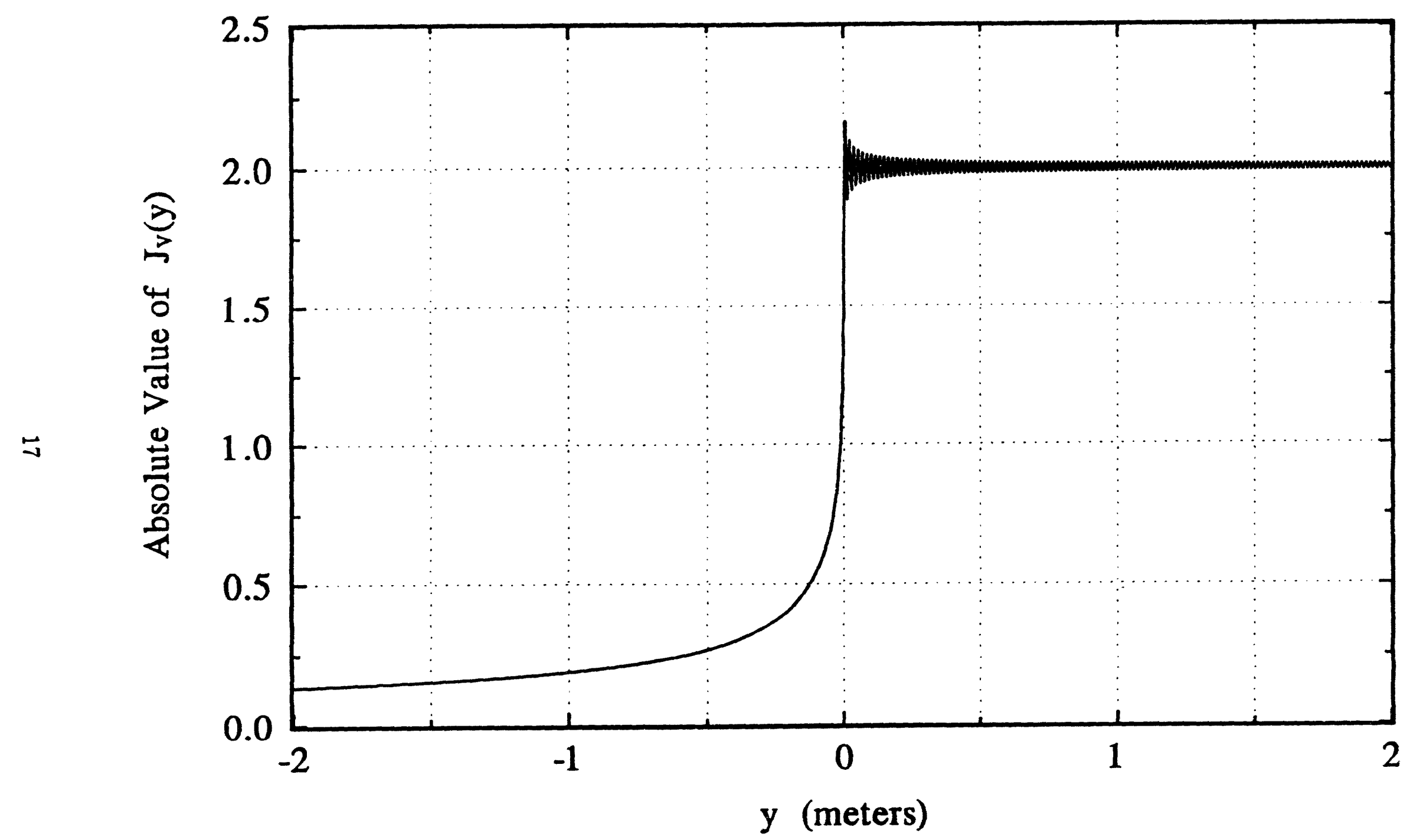

Figure 4. V-pol surface current for a wedge with smoothed edge $R=\lambda / 16$. 


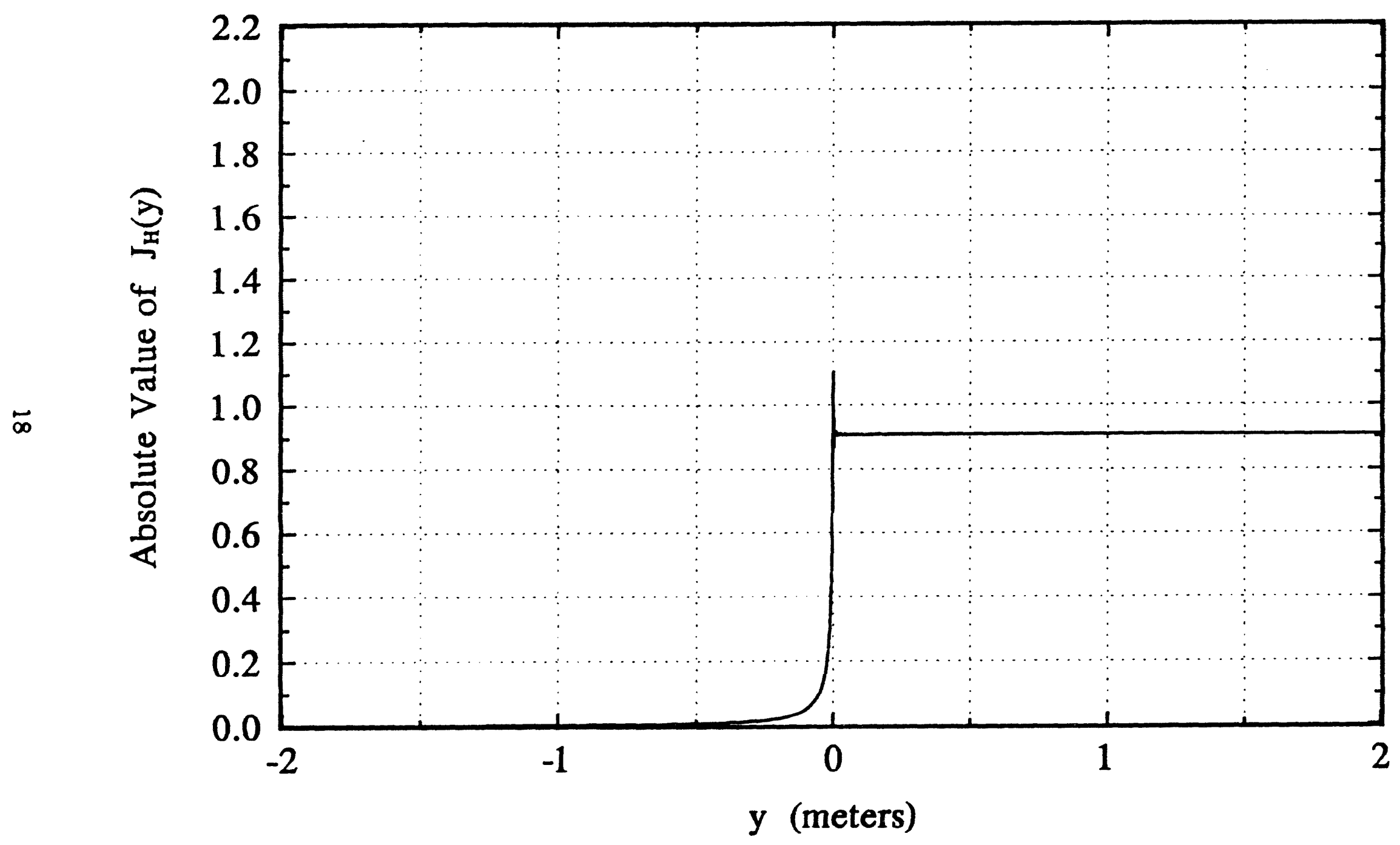

Figure 5: H-pol surface current for a wedge with smoothed edge $R=\lambda / 16$ 
$J_{V},\left|J_{V}\right|=6.75 \times 10^{-2}$. In the illuminated region, the H-pol current rapidly approaches the flat plate value while in V-pol the current has a slowly decreasing oscillating contribution in addition to the flat plate value. This occurs because $G^{V}\left(y, y^{\prime}\right)$ approaches zero more slowly than $G^{H}\left(y, y^{\prime}\right)$ as $y \Rightarrow \pm \infty$ for fixed $y^{\prime}$.

In Reference 11, expressions are derived for the calculation of the boundary conditions for the electromagnetic fields for a perfectly conducting surface. These relationships serve as important numerical checks on the calculated currents. For perfect conductors, $\mathbf{n} \cdot \mathbf{B}$ should vanish, which is non-trivial in H-pol. Figure 6 shows the absolute value of $\mathbf{n} \cdot \mathbf{B}$ employing the surface current calculated for $R=\lambda / 16$; note that $|\mathbf{n} \cdot \mathbf{B}|$ is typically much less than one percent. The only exception occurs in the vicinity of the edge, where $|\mathbf{n} \cdot \mathbf{B}|$ is slightly greater than one percent. The suggestion is that the calculated current near the edge could be in slight error, probably due to under sampling (the transition from one flat plane to the other occurs in only five points, even at a samplying of $1 / 2 \mathrm{~mm}$ ). Overall, the results shown in Figure 6 indicate that the H-pol current has been calculated quite accurately. The other boundary condition in $\mathrm{H}$-pol, the vanishing of the tangential electric field, does not lead to a new independent relationship as it is simply related to the $\mathbf{n} \cdot \mathbf{B}$ condition (see Ref. 11). As for V-pol, the non-trivial boundary condition is the vanishing of the tangential component of the electric field [the normal component condition is related to the MFIE]. Figure 7 shows the absolute value of the tangential electric field $|\mathbf{n} \times \mathbf{E}|$. It has the same general features as Figure 6, typical values less than one percent with peak values in the vicinity of the edge. The V-pol current is in slight error near the edge, but the overall accuracy is very good.

We have also shown, as one would expect from the above discussion, that the computed values for $J^{V}(y)$ and $J^{H}(y)$ are in good agreement with the Sommerfeld results. 


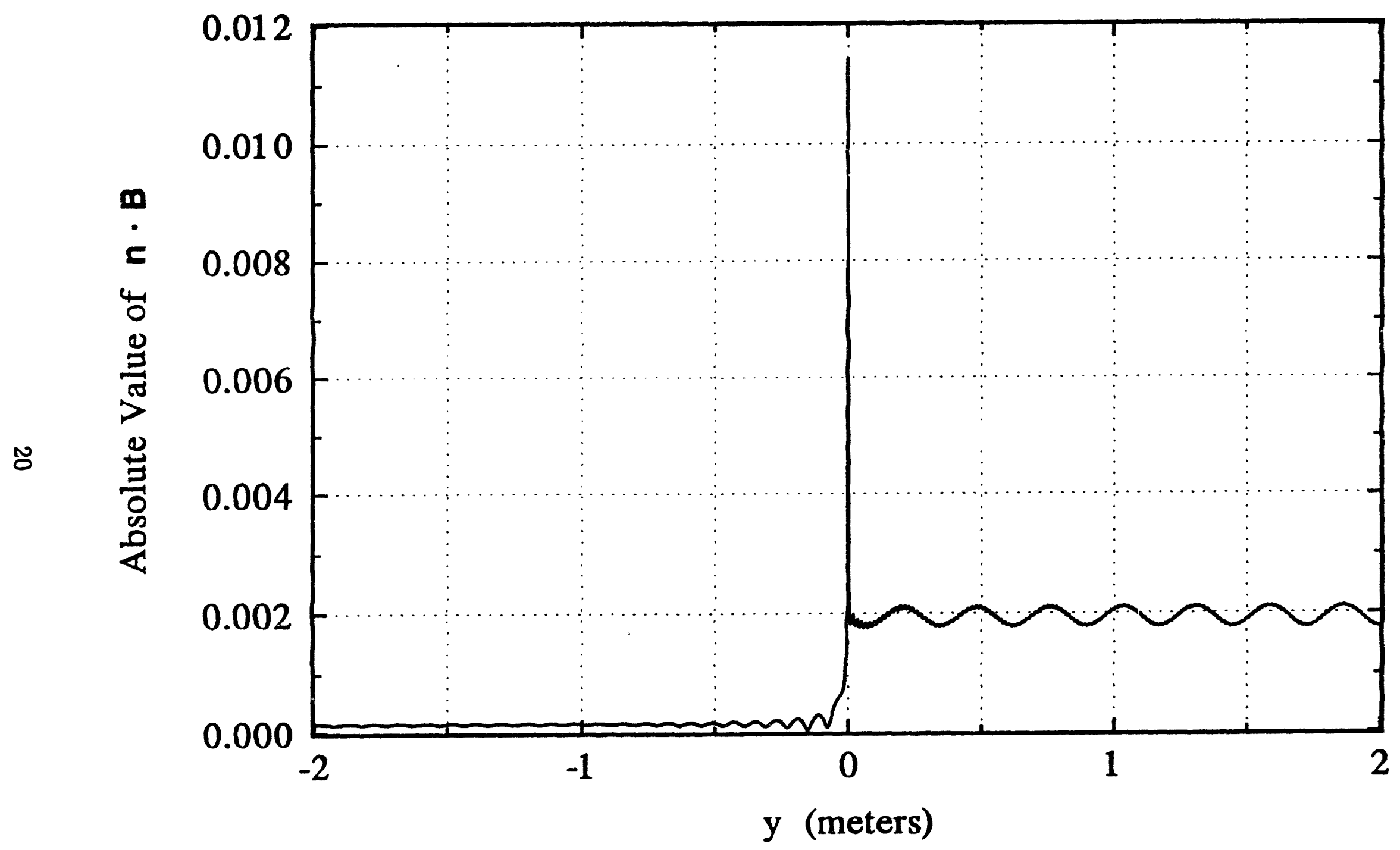

Figure 6: Boundary conditions (H-pol) for a wedge with smoothed edge $R=\lambda / 16$. 


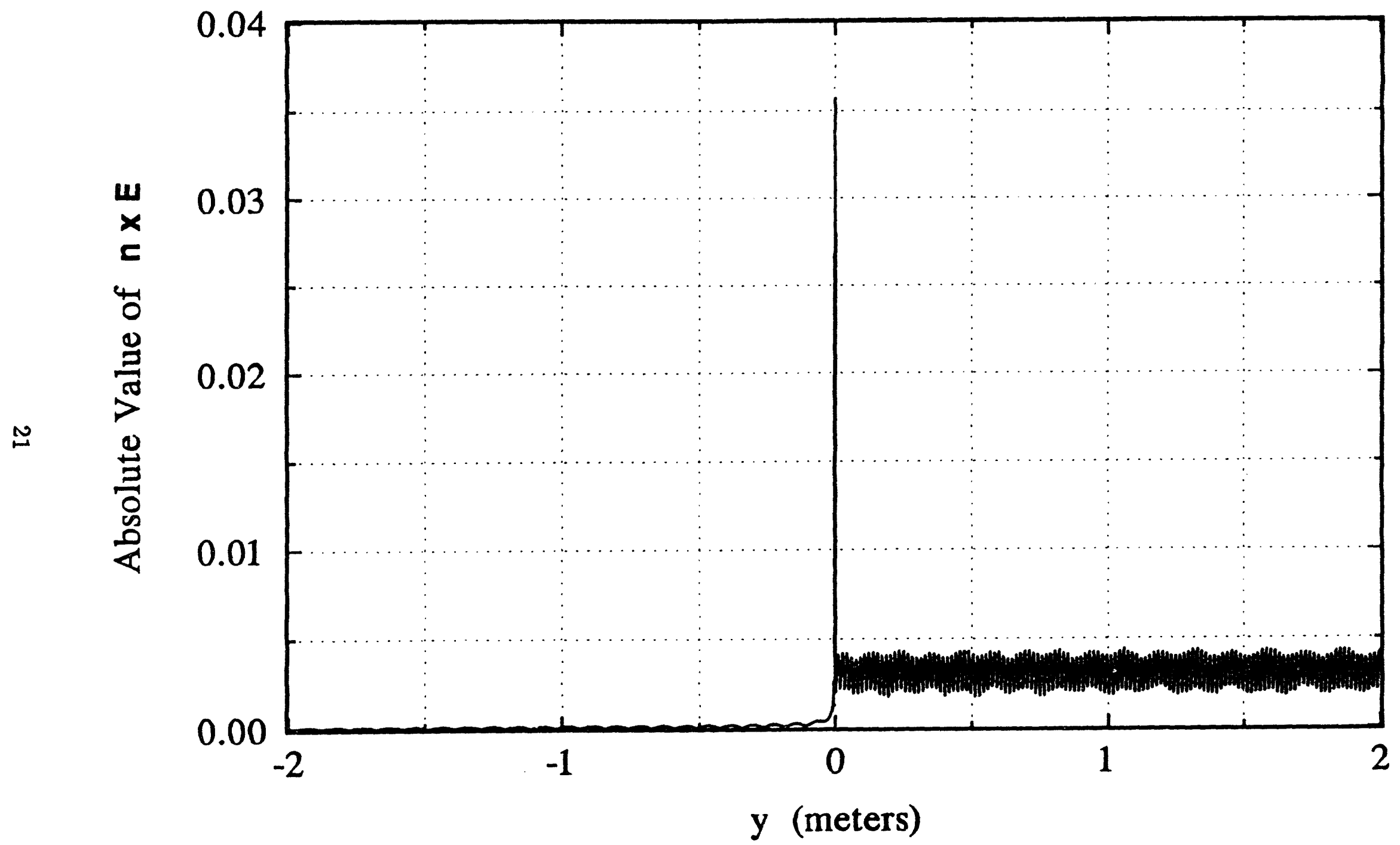

Figure 7: Boundary conditions (V-pol) for a wedge with smoothed edge $R=\lambda / 16$. 


\section{SECTION 4 \\ THE JVG WEDGE}

Reference 5, which we refer to as JVG, discusses scattering from a 3-dimensional wedge of the form

$$
\eta(x, y)=c \exp \left(-a|y|-b x^{2}\right)
$$

where

$$
\begin{aligned}
& a=0.386 \mathrm{~cm}^{-1} \\
& b=0.0166 \mathrm{~cm}^{-2} \\
& c=1.04 \mathrm{~cm} .
\end{aligned}
$$

The polarization ratio $\sigma_{H H} / \sigma_{V V}$ is reported to be about $-22 \mathrm{~dB}$ at an incidence angle of 85 degrees when the $x$-axis is perpendicular to the plane of incidence. The geometry in the vicinity of the edge $(y=0)$ is essentially the same as discussed in Sections 2 and 3. Recall that the polarization ratio for the Sommerfeld wedge was $-21.8 \mathrm{~dB}$.

We consider scattering for the one dimensional version of Eq. (55), namely,

$$
\eta(y)=c \exp (-a|y|)
$$

which has the Fourier Transform

$$
\tilde{\eta}(k)=\frac{2 a c}{k^{2}+a^{2}}
$$

To control the edge discontinuity, we define a series of filtered "wedges"

$$
\eta\left(y ; k_{\max }\right)=\frac{1}{\pi} \int_{0}^{k_{\max }} d k \tilde{\eta}(k) \cos k y .
$$

Recall that at 85 degrees incidence and for X-band, the Bragg wavenumber for backscatter is $419 \mathrm{~m}^{-1}$, so that we would expect a significant increase in the backscatter as $k_{\max }$ increases beyond $419 \mathrm{~m}^{-1}$.

The formulation to derive the currents with the iteration method is the same as that presented in Section 3 except for a few minor changes. In particular, for large negative $y$ 
(cf. Eq. (34)), we assume

$$
\begin{aligned}
& J^{V}(y)=2, \\
& y<y_{-}: \quad J^{H}(y)=2 \cos \theta,
\end{aligned}
$$

which leads to additional terms in the integral equations. In Equations (36) and (37), we have

$$
\begin{aligned}
& T_{V}(y) \Rightarrow T_{V}(y)+Z_{V}(y), \\
& T_{H}(y) \Rightarrow T_{H}(y)+Z_{H}(y),
\end{aligned}
$$

- where

$$
\begin{aligned}
& Z_{V}(y)=\int_{-\infty}^{y_{-}} d y^{\prime} \frac{1}{\widetilde{\gamma}} H_{1}(\kappa \tilde{\gamma}) e^{-i \kappa \sin \theta\left(y^{\prime}-y\right)} \\
& Z_{H}(y)=\kappa \int_{-\infty}^{y_{-}} d y^{\prime} \frac{y^{\prime}-y}{\tilde{\gamma}} H_{1}(\kappa \tilde{\gamma}) e^{-i \kappa \sin \theta\left(y^{\prime}-y\right)} .
\end{aligned}
$$

Since we are considering a low grazing angle, $T_{V}(y)$ and $T_{H}(y)$ are evaluated by means of the expressions given in Reference 11. As for $Z_{V}(y)$ and $Z_{H}(y)$, asymptotic expansions similar to Equations (41) and (42) can be derived. In particular, $Z_{V}(y)$ and the negative of $Z_{H}(y)$ can be obtained from Equations (41) and (42) by the substitutions

$$
\begin{aligned}
\sin \theta & \Rightarrow-\sin \theta, \\
u & \Rightarrow \kappa\left(y-y_{-}\right) .
\end{aligned}
$$

For each value $k_{\max }$ that we have considered, we have run three sets of calculations to check the sensitivity to the computational domain and the sampling:
(a) $y_{+}=8 \mathrm{~m}$
$y_{-}=-8 \mathrm{~m}$
$\Delta y=1 \mathrm{~mm}$
$y_{+}=4 \mathrm{~m}$
$y_{-}=-4 \mathrm{~m}$
$\Delta y=\frac{1}{2} \mathrm{~mm}$
(c) $y_{+}=4 \mathrm{~m}$
$y_{-}=-4 \mathrm{~m}$
$\Delta y=1 \mathrm{~mm}$

In each case, except for $k_{\max }=100 \mathrm{~m}^{-1}$, the backscattered field Eq. (14) in each channel changes little. The H-pol results typically change by less than a few percent while the $\mathrm{V}$-pol results tend to decrease by up to 20 percent for the longer computational domain (a). 
The Rice result (linear Bragg resonant backscatter) for the JVG wedge should be noted for comparison. When $J_{1}(y)$ found from Equation (52) is substituted into Equation (14) and the term linear in $\eta(y)$ is separated out, we obtain

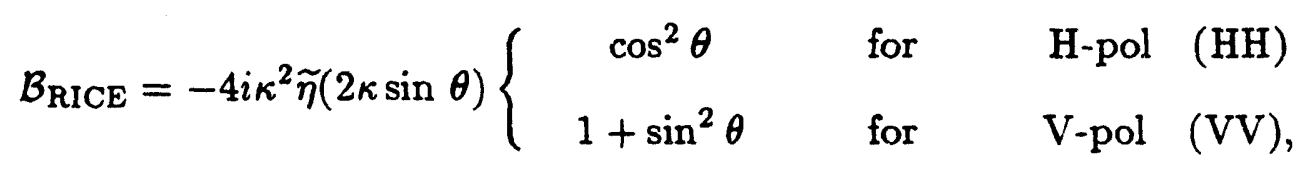

where $\tilde{\eta}(k)$ is given by Equation (58).

Table 4 shows the backscattered field $|\mathcal{B}|$ computed from Equation (14) for a series of $k_{\max }$ for parameter set (a), as well as the maximum value of the curvature. The Rice result is shown for comparison. The convergence of the iteration process is similar to that for the wedge discussed in Section 3, although the $10^{-6}$ limit occurs typically after fewer iterations. The Table indicates that for both polarizations, the backscattered fields, as expected, are rather low when $k_{\max }$ is below Bragg and quickly saturate once $k_{\max }$ is above Bragg. The saturated polarization ratio is about $-42 \mathrm{~dB}$, about $20 \mathrm{~dB}$ lower than predicted by Sommerfeld wedge scattering. Recall, all these calculations are for X-band $(\lambda=3 \mathrm{~cm})$. It is undoubtedly true that if the frequency was raised sufficiently, the wedge results of Section 3 would be approached, assuming that the geometric size of the scatterer remained the same. However, for the actual case of interest, the scatterer is so small on the scale of the incident wavelength that its "wedgelike" character is of little significance.

Table 4: Scattered Fields

\begin{tabular}{|c|c|c|c|}
\hline $\begin{array}{c}\mathbf{k}_{\max } \\
\left(\mathbf{m}^{-1}\right)\end{array}$ & $\begin{array}{c}\text { Maximum } \\
\text { Curvature } \\
\left(\mathbf{m}^{-1}\right)\end{array}$ & $\mathbf{V}$ & $\mathbf{H}$ \\
\hline 100 & 13.7 & $2.32 \times 10^{-3}$ & $7.72 \times 10^{-5}$ \\
200 & 37.5 & $3.10 \times 10^{-2}$ & $1.06 \times 10^{-3}$ \\
400 & 87.7 & 1.248 & $1.427 \times 10^{-2}$ \\
600 & 138.5 & 2.220 & $1.647 \times 10^{-2}$ \\
800 & 189.4 & 2.348 & $1.806 \times 10^{-2}$ \\
1000 & 240.4 & 2.393 & $1.870 \times 10^{-2}$ \\
2000 & 495.8 & 2.446 & $1.952 \times 10^{-2}$ \\
RICE & - & 1.598 & $6.093 \times 10^{-3}$ \\
\hline
\end{tabular}


Figures 8 and 9 show the values of $\mathbf{n} \cdot \mathbf{B}$ for H-pol and $\mathbf{n} \times \mathbf{E}$ for V-pol, respectively, for $k_{\max }=1000 \mathrm{~m}^{-1}$ and a horizontal sampling interval of $\frac{1}{2} \mathrm{~mm}$. While most of the values are very small, indicating good agreement with the perfect conductor boundary condition, the largest deviations occur at the peak $(y=0)$ of the waveform. These results indicate, not surprisingly, that very fine sampling is required in the vicinity of any region where the slope is changing rapidly. 


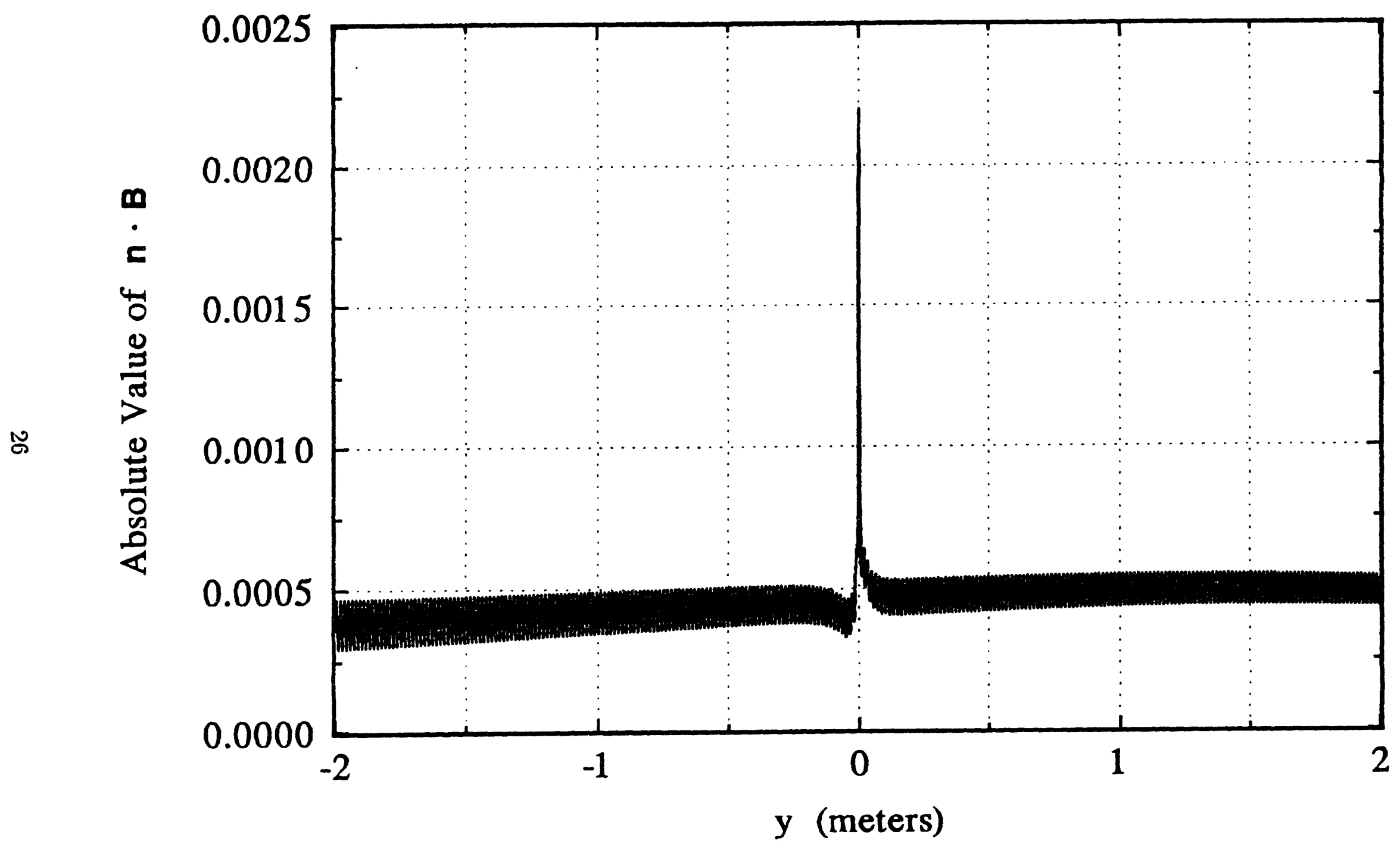

Figure 8: Boundary conditions (H-pol) for the JVG wedge with $k_{\max }=1000 \mathrm{~m}^{-1}$ and $\delta y=0.5 \mathrm{~mm}$. 


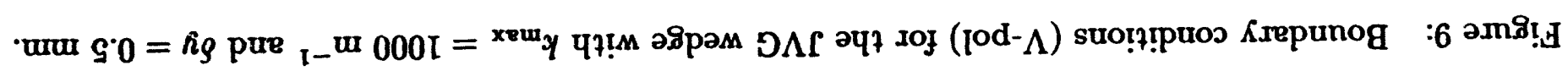

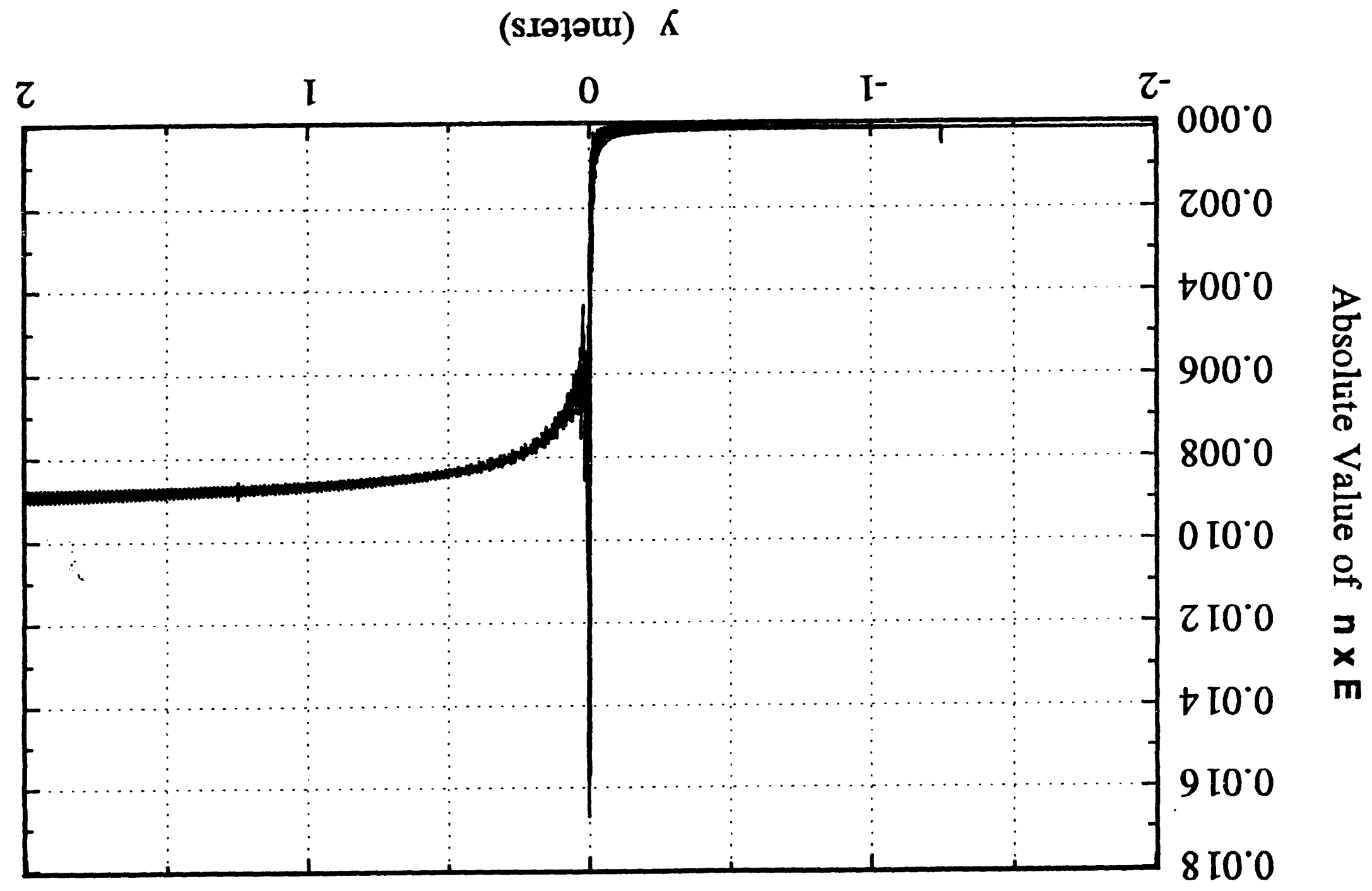




\section{SECTION 5 \\ SUMMARY OF CONCLUSIONS}

Our study of the applicability of the method of iteration to scattering from perfectly conducting wedge-like surfaces has so far led to several important conclusions:

1. Iteration of the surface current integral equation produces accurate solutions both for the Sommerfeld wedge and for the JVG "wedge".

2. The X-band backscatter polarization ratio $\left(\sigma_{H H} / \sigma_{V V}\right)$ for the JVG "wedge" at $85^{\circ}$ incidence is calculated to be $-42 \mathrm{~dB}$ rather than the $-22 \mathrm{~dB}$ reported in Reference 5; $-22 \mathrm{~dB}$ is, not coincidentally, also the ratio for the Sommerfeld wedge, the similarity of which to the JVG wedge is the implicit underlying assumption of Reference 5.

3. Consequently, backscatter from perfectly conducting truncated wedges does not appear to provide a satisfactory explanation so far for the polarization ratios observed in ocean radar return at low grazing angles. 


\section{SECTION 6 LIST OF REFERENCES}

1. Sommerfeld, A., "Mathematische Theorie der Diffraction," Math. Ann., 47, 1896, 317-374.

2. Bowman, J. J., Senior, T. B. A., and Uslenghi, P. L. E. (eds.), Electromagnetic and Acoustic Scattering by Simple Shapes, Chap. 6, "The Wedge," North-Holland Publishing Company, Amsterdam, Netherlands, 1969.

3. McNamara, D. A., Pistorius, C. W. I., and Malherbe, J. A. G., Introduction to the Uniform Geometrical Theory of Diffraction, Chap. 4, "Two-Dimensional Wedge Diffraction," Artech House, Boston, MA, 1990.

4. Lyzenga, D. R., Maffètt, A. L., and Shuchman, R. A., "The Contribution of Wedge Scattering to the Radar Cross Section of the Ocean Surface," IEEE Transactions on Geoscience and Remote Sensing, GE-21, ㄴ, October 1983.

5. Jensen, G. A., Vesecky, J. F., and Glazman, R. E., "Diffraction from Sharply Peaked Waves as an Ocean Surface Scattering Mechanism," IGARSS 'g2, II, May 1992, 1771-1773.

6. Long, M. W., Radar Reflectivity of Land and Sea, Artech House, Boston, MA, 1983, 286-287.

7. Oberhettinger, F., "On Asymptotic Series for Functions Occurring in the Theory of Diffraction of Waves by Wedges," J. Math. Phys., 34, 1956, 245-255.

8. Keller, J. B., "Geometrical Theory of Diffraction," J. Opt. Soc. of America, 52, February 1962, 116-130.

9. Kouyoumjian, R. G., and Pathak, P. H., "A Uniform Geometrical Theory of Diffraction for an Edge in a Perfectly Conducting Surface," Proc. IEEE, 62, November $1974,1448-1461$.

10. Knott, E. F., and Senior, T. B. A., "Comparison of three high-frequency diffraction techniques," Proc. IEEE, 62, 1974, 1468-1474.

11. Holliday, D., DeRaad, L. L., and St-Cyr, G. J., "Electromagnetic Scattering from Smooth Ocean-Like Surfaces at Low Grazing Angles," RDA-TR-1870001-001, Logicon $\mathrm{R} \& \mathrm{D}$ Associates, Los Angeles, CA, April 1993 (Submitted to IEEE Transactions on Antennas and Propagation). 
11
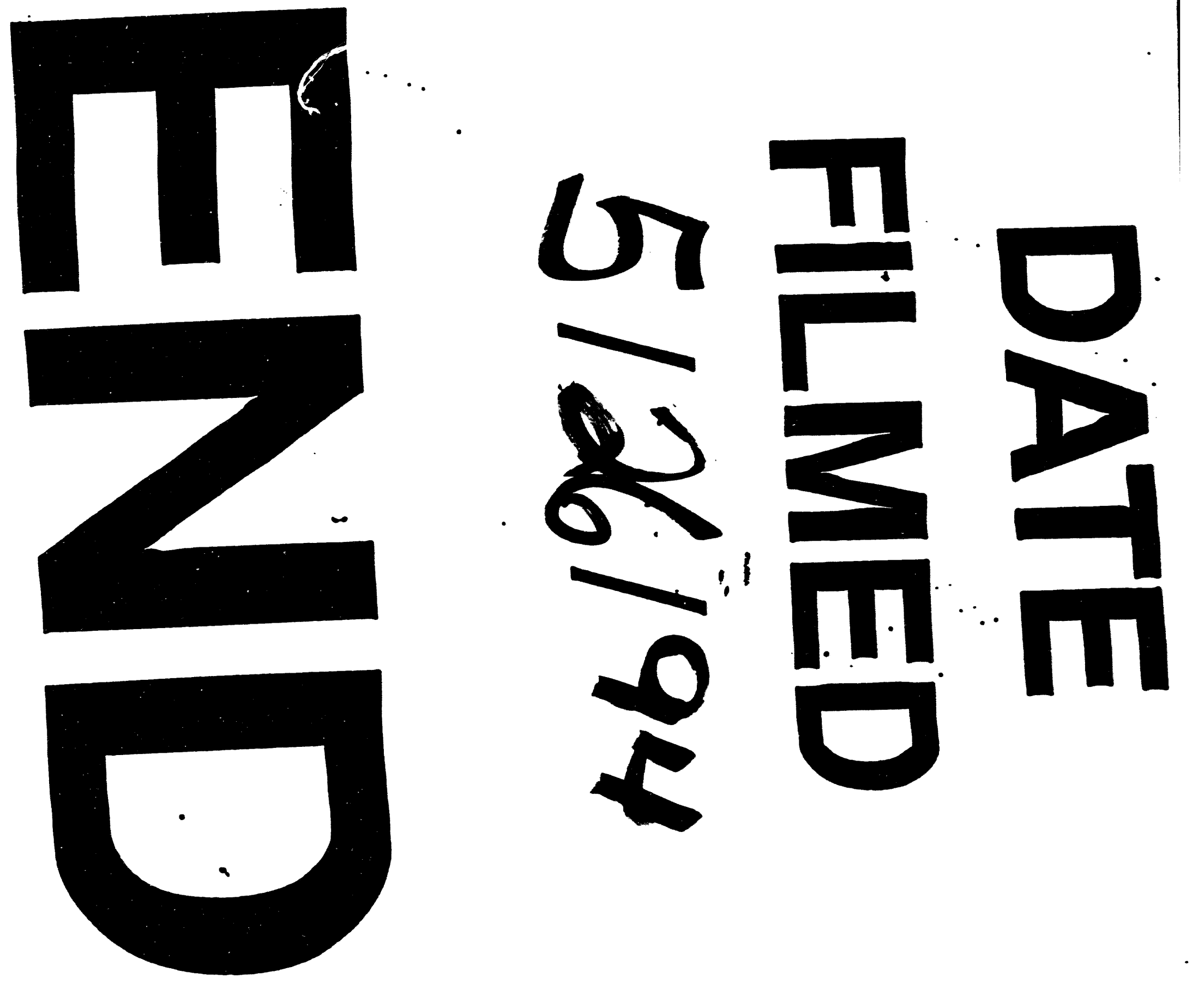

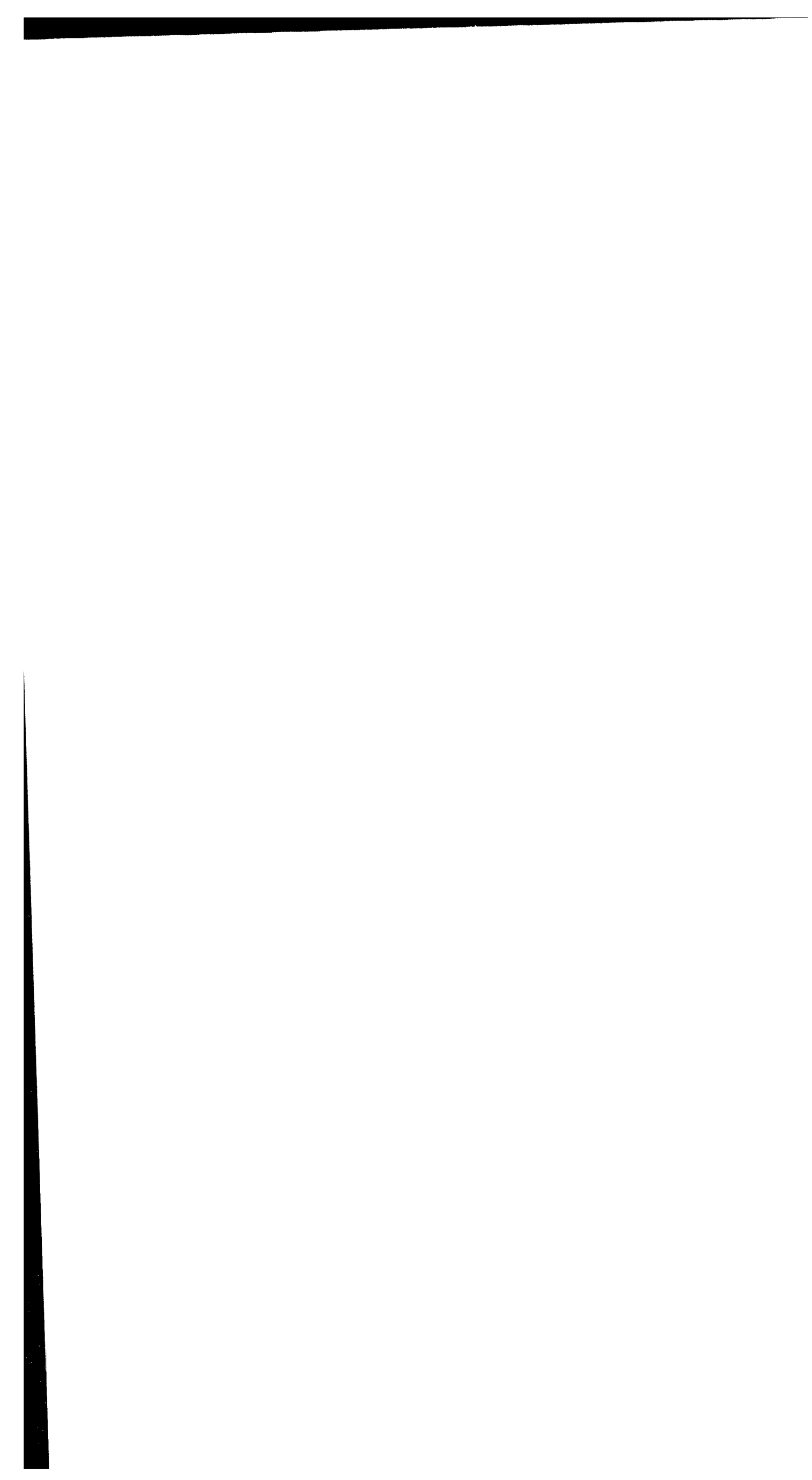